Article

\title{
Adolescents' Peer Friendship and Anxiety and Depression among First-Generation Immigrant BAME Families in the UK
}

\author{
Juan Du ${ }^{1, *}$ and Andy P. Field ${ }^{2}$ (D) \\ 1 Metanoia Institute, Middlesex University, London W5 3XD, UK \\ 2 School of Psychology, University of Sussex, Brighton BN1 9QH, UK; andy.field@sussex.ac.uk \\ * Correspondence: juan.du@metanoia.ac.uk
}

Received: 31 March 2020; Accepted: 20 May 2020; Published: 29 May 2020

\begin{abstract}
There is equivocal evidence on how being a child in a Black, Asian and Minority Ethnic (BAME) immigrant family affects internalizing symptoms such as anxiety. This cross-sectional study examined the relationships between peer friendships and anxiety/depression symptoms in BAME immigrant adolescents (IA) and white native adolescents (WNA). Method: Sixty-two adolescents from the UK ( $\mathrm{IA}=26$, WNA $=36$, mean age $=14$ years $)$ were assessed on close friendship, social competence, social anxiety, and depression. Immigrant family parents $(n=23)$ were also assessed on cultural orientation. There were no significant differences in anxiety and depression between groups. Bayes factors supported the conclusion that the groups did not differ. However, IA and WNA groups had different patterns of associations between close friendship/social competence and anxiety and depression symptoms. Close friendships were more strongly associated with lower anxiety/depression in IAs than WNAs, and social competence was more strongly associated with lower anxiety/depression in WNAs than IAs. Moderation analyses indicated that the relationship between close friendship and social and separation anxiety was significantly moderated by ethnic group, as was the relationship between social competence and generalized anxiety. The findings suggest that social and separation anxiety are more strongly associated with close friendships for BAME immigrant children than for non-immigrant adolescents. As such, activities that help BAME immigrant children to foster close relationships may have positive effects on their well-being.
\end{abstract}

Keywords: peer friendships; social anxiety; BAME adolescents

\section{Introduction}

Adolescence is a critical period in psycho-social development, often marked by expansion of peer social networks, increased closed friendships and romantic relationships (La Greca and Harrison 2005). Social anxiety, characterized by a persistent and intense fear of being judged by others, is one of the most common anxiety disorders for adolescents, with the average early onset age of 13 years (National Institute for Health and Care Excellence 2013) and a lifetime prevalence of around $12 \%$ of the general population (Kessler et al. 2005). Social anxiety not only affects adolescents' academic learning and social development (Cartwright-Hatton et al. 2011), but persisting social anxiety is comorbid with depression later in life (Beesdo et al. 2007).

For first-generation Black, Asian and Minority Ethnic (BAME) immigrant adolescents-children and parents who were born abroad and whose first language is not the host language (Perreira and Ornelas 2011) - the social landscape is complicated: not only do they face the usual challenges of adolescents, but also have to negotiate between different cultures and explore whether they feel a sense of belonging in the host society (Lee 2019), the additional stresses of adjusting to a bicultural setting, 
learning a new language, finding new friends, and facing anti-immigration sentiments (Louise and Stalford 2004) and experiencing racial discrimination (Seaton et al. 2009). Qin et al. (2008) illustrate such difficulties in Asian American families, where they argue that immigrant parents' lack of knowledge of the host culture and English proficiency has a negative impact on their children's wellbeing; for example, children who have superior language ability to their parents might have the burden of acting as a translator. This unique situation could have important practical implications for the adolescent's functioning at school, and within the community and society more widely (Woolcock and Narayan 2000).

Surprisingly, relatively few studies have investigated immigrant adolescents' psychosocial adjustment and what variables moderate this adjustment. Despite the huge knowledge gap in the literature, the existing evidence is contradictory, supporting all possibilities of differences between immigrant adolescents and their native counterparts on measures of social wellbeing and anxiety: compared to native children, children from immigrant families (1) have worse psychological wellbeing (notably internalizing problems); (2) have better psychological wellbeing; (3) do not differ in psychological wellbeing. We will review each possibility in turn.

Firstly, some studies suggest that immigrant children are more likely to report symptoms associated with psychological disorders (Bhugra 2004; Louise and Stalford 2004; Qin et al. 2008; Huang et al. 2012). A Danish study also showed that post-migration discrimination experience is associated with increased internalized problems, such as anxiety (Montgomery 2008). However, this study also showed that immigrant children's internalized problems were reduced once they had good peer friendships with, for example, a higher number of native friends.

Although the aforementioned studies indicate that immigrant children have greater internalizing problems than native children, a US national survey for adolescents (aged 12-18) found the opposite: immigrant children scored lower than native (US born) adolescents on depression and higher on positive wellbeing (Harker 2001).

Finally, there is evidence that wellbeing in native and immigrant adolescents is very similar. For example, Atzaba-Poria et al. (2004) compared primary school children of both Indian and English origins in the UK. Anxiety, social withdrawal, depression and behavioural problems were measured using parental report, whereas children self-reported their self-worth and friendship quality. The results were similar across the groups. Similarly, a cross-sectional study by Pina and Silverman (2004) compared clinically anxious adolescents from European American backgrounds to Hispanic/Latinos and found that, at the group level, the immigrant and native's anxiety levels were more similar than different. However, significant differences emerged when a native language assessment was used.

There is equivocal evidence of how being a child in an immigrant family affects internalizing symptoms such as anxiety. A likely explanation for the inconsistent evidence is the presence of moderating variables. Several variables have been suggested as psychologically protective factors in immigrant children: the family's social position and economic status (Moitra et al. 2014; Blinder et al. 2011), parental host language proficiency and parents' cultural integration (Qin et al. 2008), the child's social peer relationships and social support (Kovacev and Shute 2004), parents' supervision and social support (Harker 2001), immigrant parents' education level and ambitions, desire for a better life (Hao and Woo 2012). All of these variables could feasibly protect immigrant children from internalizing symptoms, but peer relationships, in particular, are a strong candidate because of adolescents' strong desire to accepted by their peers, belong to a group, and to share feelings, values and activities with close friends (La Greca and Harrison 2005). In general, peer relationships are an important moderator of emotional and social development (Sirin et al. 2013). For example, good quality friendships can buffer victimization (Cardoos and Hinshaw 2011) and having trusted and close friends can protect socially anxious adolescents from facing anxiety-provoking situations (Van Zalk and Zalk 2014). Likewise, having positive social support, such as friends' acceptance and validation, is associated with lower levels of social anxiety (Festa and Ginsburg 2011). Conversely, problematic peer-relationships and lack of friendship can act as causal risk factors for anxiety (Rapee and Spence 2004), and a lack of positive peer friendship has been linked to social mistakes and impaired understanding of social blunder (Banerjee et al. 2011). 
In summary, there is conflicting evidence for the impact of being a BAME immigrant child on psychological wellbeing and social anxiety. This inconsistency is likely due to the presence of moderations such as peer relationships. Therefore, this study is important and unique as, based on our limited research, this is the first study to investigate the understanding of peer friendships among BAME immigrant adolescents' social anxiety in the UK via comparison with their native white peers. Hence, the primary aim of this study is to compare anxiety symptoms between the two groups and to establish whether peer relationships moderate anxiety differences between within these groups. We hypothesize that immigrant children with poorer peer relationships will experience greater anxiety than native children with comparably poor peer relationships, conversely, where peer relationships are good, differences between immigrant and non-immigrant families will be minimized.

\section{Method}

\subsection{Participants}

Sixty-eight adolescents were recruited. Two participants were excluded because of large numbers of incomplete responses. One participant was excluded as a statistical outlier. Three participants from mixed families (i.e., an adolescent who had one parent born in the UK, with the other born outside of the UK) were also excluded from the study.

The final sample included data from 62 adolescents ( 26 girls, 36 boys) aged from 10 to 16 years old $(M=14, S D=1.23)$. The IA group $(n=26)$ consisted of first-generation immigrant adolescents (i.e., BAME adolescents and parents were born outside of the UK and their first domestic language was non-English); the WNA group ( $n=36$ ) consisted of non-immigrant families (i.e., children born in the UK, and their first domestic language was English). From the 26 immigrant families, 23 parents responded to the parental culture orientation questionnaires. All adolescents and families were currently living in one of four different areas of England (Surrey, Sussex, Hertfordshire, and Buckinghamshire). Due to time constraints, participant recruitment was through snowball sampling and only four secondary schools from these four areas agreed to participate in this study. From these, $89 \%(n=55)$ of the participants were recruited from schools; $11 \%(n=7)$ of the sample were recruited through community connections or were self-referred responses to social media advertisements. The response rate from schools was $30 \%$ for immigrant families and $55 \%$ for non-immigrant families.

The racial and ethnic background profile of the participants was $58.1 \%(n=36)$ English; $12.9 \%$ $(n=8)$ Chinese; $6.5 \%(n=4)$ African origin; $6.5 \%(n=4)$, Afro Romanian; $9.7 \%(n=6)$ from South Asia (Pakistan); $6.5 \%(n=4)$ from Middle East-Arabic.

\subsection{Measures}

\subsubsection{The Revised Children's Anxiety and Depression Scale (RCADS)}

The RCADS (Chorpita et al. 2000) includes 47 items using a 4-point Likert-Scale (never = 0, always =3), with subscales of social anxiety, depression, generalized anxiety disorder, obsessive-compulsive disorder, panic disorder, separation anxiety. The internal consistencies for this scale were: social anxiety (nine items) Cronbach' $\alpha=0.86$; generalized anxiety (six items), $\alpha=0.80$; depression (10 items), $\alpha=0.92$; separation anxiety (seven items), $\alpha=0.80$; and panic disorder (nine items), $\alpha=0.90$.

\subsubsection{Self-Perception Profile for Adolescents Questionnaire-What I am Like (SPPA)}

The SPPA scale (Harter 2012) has 45 items, including nine subscales, each with five items, and a 4-point response scale. We used only the social acceptance and close friendships subscales. Higher scores reflect greater perceived competence. For each item, two sentences are presented side by side, and participants decide which sentence best describes them and indicate whether the statement was "sort of true" or "really true". For the close friendship subscale, an example of the two sentences is: "Some teenagers are able to make really close friends" vs. "Other teenagers find it hard to make 
really close friends". Internal consistencies were high for both the close friendship subscale, $\alpha=0.76$, and social competence subscale, $\alpha=0.82$.

\subsubsection{The Adapted General Ethnicity Questionnaire (AGEQ)}

The AGEQ (Tsai et al. 2000) is aimed at parents from immigrant families, and consists of 38 items with a 5-point Likert response scale. The questionnaire measures domains of culture including language use, engagement in cultural practices, social affiliation, and cultural identification. We used the scale total as an overall gauge of engagement with British culture, which yielded a high internal consistency, $\alpha=0.93$.

\subsection{Procedure}

This study employed two recruitment routes: through three state secondary schools or via community-based and social media advertisements. In schools, information sheets were sent out to parents either by mail or directly to the pupils. The families who returned two signed consent forms (from both adolescents and parents) were given paper and pencil surveys to complete in a quiet classroom, with both the teacher and researcher present at the time of completion. The same recruitment information letter was used to recruit from the community. The information sheet and consent forms were then either posted or emailed to interested parties responding to the adverts. The questionnaire packs were sent out either by letter with pre-paid return envelopes, emails, or face-to-face.

Prior to beginning the study, all participants were given instructions on how to fill in the questionnaires. They were informed that there were no right or wrong answers and encouraged to answer the questions honestly. Participants could skip any items they did not want to answer, or they could ask for assistance. At the end of survey, participants were given the opportunity to ask questions and were fully debriefed. The researcher also provided information signposting supporting charities for adolescents who expressed feeling anxious.

Parents from the IA $(n=23)$ completed the AGEQ survey. In case of limited English language proficiency in parents, an option was given for the adolescents to fill in the questionnaire on behalf of their parents. However, all participants completed the questionnaires in English, except one parent from a Chinese immigrant family, who wished to complete the questionnaires in Chinese. As the researcher's first language is Chinese, accurately translated forms were provided to facilitate this request.

\section{Results}

\subsection{Differences between IA and WNA}

Table 1 presents Welch two-sample $t$-tests comparing WNA and IA groups on all study variables computed using R (R Core Team 2015). None of the differences were significant. Because these tests could be underpowered, Bayes Factors $\left(\mathrm{BF}_{10}\right)$ were computed for each comparison, which quantifies the ratio of the probability of the data under the alternative hypothesis relative to the null. A value of 1 , therefore, means that the observed data are equally probable under the null and alternative hypotheses; values below 1 suggest that the data are more probable under the null hypotheses relative to the alternative. Bayes factors provide information about the probability of the data under the null hypothesis (relative to the alternative), whereas significance tests provide no evidence at all about the status of the null hypothesis (Dienes 2014).

Bayes Factor was estimated using the BayesFactor (Morey and Rouder 2014) package in R, which uses a default Jeffries prior (Rouder et al. 2009), which models prior beliefs in the effect size using a Cauchy distribution centered on 0 and with a default scale factor of 0.707 . In doing so, our prior belief is that there is a $50 \%$ probability that the effect size $(d)$ lies between -0.707 to 0.707 . This default value represents a fairly open-minded belief that effects could range from fairly large and positive in the predicted direction to equally large in the opposite direction. All of the Bayes Factors (Table 1) suggest 
much stronger evidence for the null hypotheses, except for the close friendship subscale, where there was roughly equal evidence for the null and alternative hypothesis.

Table 1. Summary of study variable of means, standard deviations and mean difference, $95 \% \mathrm{CI}, t$-value, $p$ value, effect size.

\begin{tabular}{|c|c|c|c|c|c|c|c|c|c|c|c|}
\hline \multirow[t]{2}{*}{ Variable } & \multicolumn{2}{|c|}{ IA $(n=26)$} & \multicolumn{2}{|c|}{ WNA $(n=36)$} & \multirow[t]{2}{*}{$\begin{array}{c}\text { Mean } \\
\text { Difference }\end{array}$} & \multicolumn{2}{|c|}{$95 \%$ CI } & \multirow[t]{2}{*}{$t$} & \multirow[t]{2}{*}{$d f$} & \multirow[t]{2}{*}{$p$} & \multirow[t]{2}{*}{$\mathrm{BF}_{01}$} \\
\hline & $M$ & $S D$ & $M$ & $S D$ & & Lower & Upper & & & & \\
\hline SPPA & & & & & & & & & & & \\
\hline Close friendship & 14.27 & 3.46 & 15.83 & 2.97 & -1.56 & -3.25 & 0.12 & -1.86 & 48.84 & 0.069 & 1.19 \\
\hline $\begin{array}{l}\text { Social } \\
\text { competence } \\
\text { RCADS }\end{array}$ & 14.62 & 3.42 & 14.92 & 2.82 & -0.30 & -1.95 & 1.35 & -0.37 & 47.46 & 0.715 & 0.28 \\
\hline Social anxiety & 48.12 & 13.23 & 46.42 & 11.65 & 1.70 & -4.81 & 8.21 & 0.52 & 49.69 & 0.602 & 0.30 \\
\hline Major depression & 51.00 & 16.50 & 52.39 & 15.90 & -1.39 & -9.78 & 7.00 & -0.33 & 52.82 & 0.741 & 0.27 \\
\hline $\begin{array}{c}\text { Generalized } \\
\text { anxiety }\end{array}$ & 44.58 & 10.30 & 45.08 & 11.89 & -0.51 & -6.17 & 5.16 & -0.18 & 57.95 & 0.859 & 0.27 \\
\hline $\begin{array}{c}\text { Separation } \\
\text { anxiety }\end{array}$ & 50.77 & 12.21 & 49.06 & 10.94 & 1.71 & -4.33 & 7.76 & 0.57 & 50.32 & 0.572 & 0.30 \\
\hline Panic Disorder & 53.58 & 15.82 & 53.00 & 19.27 & 0.58 & -8.36 & 9.51 & 0.13 & 58.94 & 0.900 & 0.26 \\
\hline
\end{tabular}

\subsection{Moderation Analysis}

The initial analysis suggests that IA and WNA groups have comparable levels of anxiety. However, our main hypothesis is that anxiety in IA adolescents would be moderated by their peer friendships, more so than WNA adolescents. This hypothesis was addressed first by looking at the correlations between social relationships and anxiety in IA (Table 2) and WNA groups (Table 3), and then by testing for moderation by predicting anxiety from peer friendships, ethnic group (IA vs. WNA dummy coded as immigrant adolescents $=0$; non-immigrant adolescents $=1$ ) and their interaction (Table 4). Analyses were conducted using Hayes (2013) PROCESS tool for SPSS (Hayes 2013).

For IA (Table 2) close friendship was significantly correlated with all study variables except parental cultural orientation: in particular, there was a significant negative correlation with social anxiety, separation anxiety, and depression. As was predicted, more positive close friendships were associated with lower anxiety and depression scores. The pattern was different for perceived social competence, which was not significantly associated with any anxiety variables (although effects are small to medium in size). There was no significant relationship between IA parents' cultural orientation and their adolescents' social anxiety, but it did have a significant negative relationship with their adolescents' depression.

For WNA (Table 3) close friendships were also significantly negative correlated with depression, but not with anxiety variables (and effect sizes were close to zero). Conversely to the IA group, social competence was significantly negatively correlated with all anxiety variables: higher social competence was associated with lower anxiety symptoms for native adolescents.

The zero-order analysis of relationships demonstrated a difference pattern between peer friendship and anxiety in IA and WNA adolescents. The moderation analyses tested whether these patterns were indeed different in IA and WNA groups by predicting each subscale of the RCADS from ethnicity, close friendships/social competence and their interaction. Table 4 summarizes the 10 moderation models. Three significant interactions were found: Ethnicity $\times$ Close friendship significantly predicted social anxiety, $t=2.85, p=0.006$ and separation anxiety, $t=2.10, p=0.04$; Ethnicity $\times$ Social competence significantly predicted generalized anxiety, $t=-2.15, p=0.036$. There were no other significant Ethnicity $\times$ Close friendship or Ethnicity $\times$ Social competence interactions. 
Table 2. Correlation matrix for study variables for BAME immigrant adolescents $(n=26)$. $95 \%$ percentile bootstrap confidence intervals in parentheses.

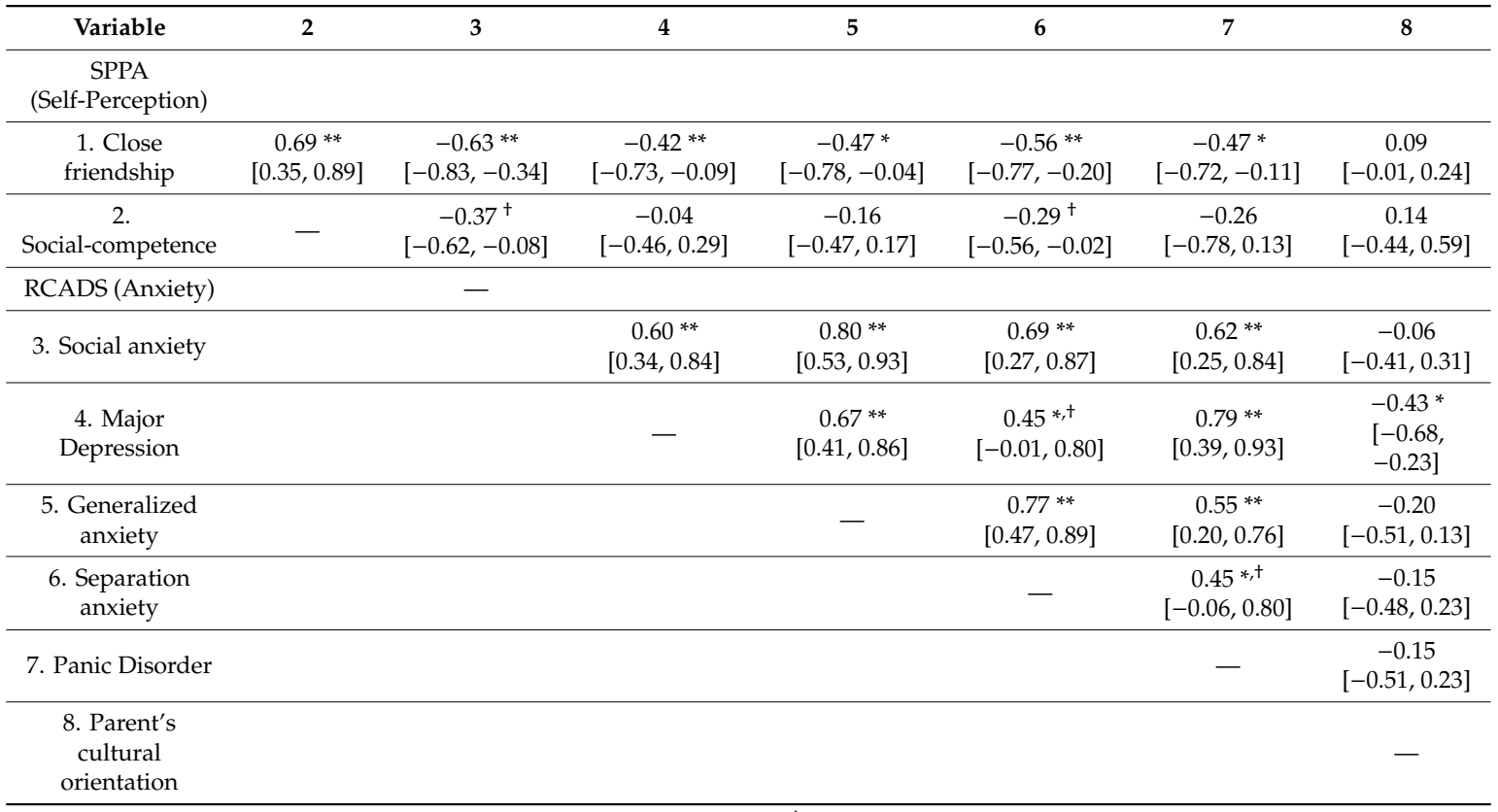

Notes: $\mathrm{ns}=$ non-significant $(p>0.05),{ }^{*} p<0.05,{ }^{* *} p<0.01 ;{ }^{+} 95 \%$ bootstrap confidence interval does not contain zero but $p>0.05$ or vice versa. Gender did not significantly moderate any of these correlations.

Table 3. Correlation matrix for all study variables for non-immigrant adolescents $(n=36)$. $95 \%$ percentile bootstrap confidence intervals in parentheses.

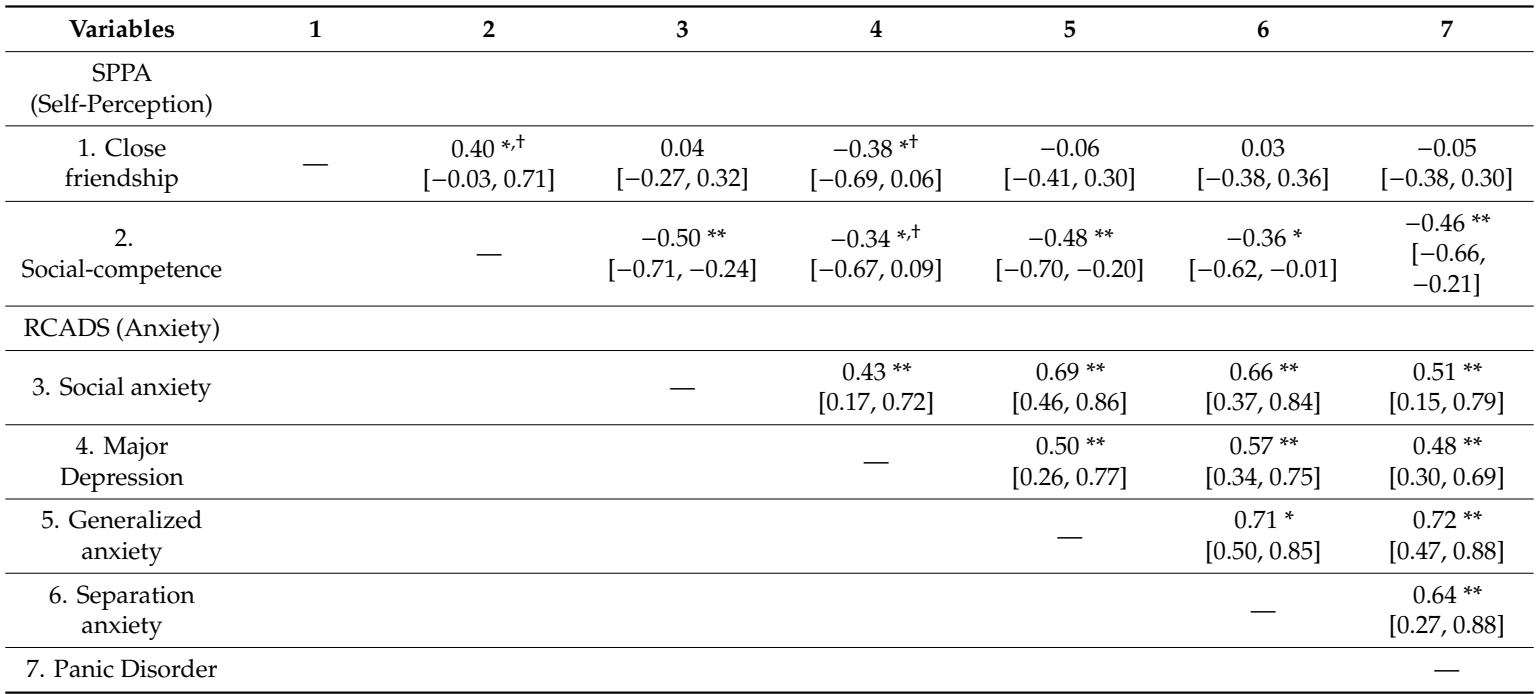

Notes: $\mathrm{ns}=$ non-significant $(p>0.05),{ }^{*} p<0.05,{ }^{* *} p<0.01 .{ }^{\dagger} 95 \%$ bootstrap confidence interval does not contain zero but $p>0.05$ or vice versa. Gender did not significantly moderate any of these correlations. 
Table 4. Parameter estimates (95\% Confidence intervals in parentheses) models testing ethnic group as a moderator of the relationship between close friendships, social competence and RCADS subscales.

\begin{tabular}{|c|c|c|c|c|c|}
\hline & Social Anxiety & Depression & $\begin{array}{c}\text { Generalized } \\
\text { Anxiety }\end{array}$ & $\begin{array}{l}\text { Separation } \\
\text { Anxiety }\end{array}$ & Panic Disorder \\
\hline \multicolumn{6}{|c|}{ Predictor: Close Friendships } \\
\hline Intercept & $\begin{array}{c}46.17^{* * *} \\
{[43.55,48.79]}\end{array}$ & $\begin{array}{c}51.82^{* * *} \\
{[47.43,56.20]}\end{array}$ & $\begin{array}{c}44.43^{* * *} \\
{[41.53,47.32]}\end{array}$ & $\begin{array}{c}48.97^{* * *} \\
{[46.39,51.54]}\end{array}$ & $\begin{array}{c}52.51^{* * *} \\
{[43.55,48.79]}\end{array}$ \\
\hline Ethnicity & $\begin{array}{c}0.32 \\
{[-4.89,5.52]}\end{array}$ & $\begin{array}{c}4.51 \\
{[-4.00,13.02]}\end{array}$ & $\begin{array}{c}1.97 \\
{[-3.59,7.53]}\end{array}$ & $\begin{array}{c}0.04 \\
{[-5.03,5.10]}\end{array}$ & $\begin{array}{c}1.65 \\
{[-6.44,9.73]}\end{array}$ \\
\hline Close Friendship & $\begin{array}{c}-0.88 \\
{[-1.76,0.01]}\end{array}$ & $\begin{array}{c}-1.99 * \\
{[-3.66,-0.34]}\end{array}$ & $\begin{array}{c}-0.75 \\
{[-1.84,0.35]}\end{array}$ & $\begin{array}{c}-0.78 \\
{[-1.81,0.26]}\end{array}$ & $\begin{array}{c}-1.11 \\
{[-2.58,0.36]}\end{array}$ \\
\hline Ethnicity $\times$ Close & $2.51 * *$ & -0.02 & 1.17 & $2.12 *$ & 1.91 \\
\hline Friendship & {$[0.75,4.28]$} & {$[-3.04,2.99]$} & {$[-0.91,3.25]$} & {$[0.09,4.14]$} & {$[-0.85,4.68]$} \\
\hline$R^{2}$ & 0.18 & 0.16 & 0.08 & 0.16 & 0.08 \\
\hline \multicolumn{6}{|c|}{ Predictor: Social Competence } \\
\hline Intercept & $\begin{array}{c}47.20 * * * \\
{[44.20,50.21]}\end{array}$ & $\begin{array}{c}1.99 * * * \\
{[47.69,56.29]}\end{array}$ & $\begin{array}{c}45.01 * * * \\
{[42.21,47.81]}\end{array}$ & $\begin{array}{c}49.82 * * * \\
{[46.89,52.74]}\end{array}$ & $\begin{array}{c}53.41 * * * \\
{[48.96,57.85]}\end{array}$ \\
\hline Ethnicity & $\begin{array}{c}-1.25 \\
{[-7.57,5.07]}\end{array}$ & $\begin{array}{c}1.53 \\
{[-7.37,10.43]}\end{array}$ & {$[-4.87,6.44]$} & $\begin{array}{c}-1.39 \\
{[-7.43,4.65]}\end{array}$ & $\begin{array}{c}-0.03 \\
{[-8.95,8.88]}\end{array}$ \\
\hline Social Competence & $\begin{array}{c}-1.64 * * * \\
{[-2.56,-0.72]}\end{array}$ & $\begin{array}{c}-0.86 \\
{[-2.58,0.85]}\end{array}$ & $\begin{array}{c}-1.21 * \\
{[-2.15,-0.26]}\end{array}$ & $\begin{array}{c}-1.16^{*} \\
{[-2.07,-0.24]}\end{array}$ & $\begin{array}{c}-2.17^{* *} \\
{[-3.61,-0.73]}\end{array}$ \\
\hline Ethnicity $\times$ Social & -1.02 & -2.51 & $-1.90 *$ & -0.56 & -2.26 \\
\hline Competence & {$[-2.87,0.84]$} & {$[-5.86,0.84]$} & {$[-3.73,-0.07]$} & {$[-2.33,1.21]$} & {$[-5.08,0.56]$} \\
\hline$R^{2}$ & 0.17 & 0.07 & 0.08 & 0.10 & 0.15 \\
\hline
\end{tabular}

Note. ns = non-significant $(p>0.05),{ }^{*} p<0.05,{ }^{* *} p<0.01,{ }^{* * *} p<0.001$.

\section{Discussion}

The current study investigated whether the BAME immigration stressor is a significant risk factor for immigrant adolescents' anxiety and depression compared to white native adolescents and examined the correlation between peer friendship and anxiety symptoms. The Bayesian analysis strongly suggested that BAME immigrant and non-immigrant adolescents did not differ in their levels of anxiety and a depression at the group level. However, as predicted, there were some differences between groups in how social relationships might act as a buffer against anxiety and depression.

The overall finding that IA and WNA groups did not differ on anxiety and depression is consistent with some of the previous literature. For example, Atzaba-Poria et al. (2004) compared anxiety levels in primary school children of British native and Indian origin and found that they were very similar. Similarly, Pina and Silverman (2004) study of immigrants of European, American and Latino backgrounds found more similarities in anxiety levels than differences. However, the finding is inconsistent with studies that show that immigrant children are less anxious and have more positive psychological wellbeing than native counterparts (e.g., Harker 2001), or those suggesting that immigrant status is associated with psychological disorders (e.g., Bhugra 2004). These differences could be due to the heterogeneity of the immigrant samples researched in different studies, and countryor ethnic-specific difficulties faced by particular immigrant groups in particular countries. However, it might also reflect the presence of moderator variables.

The moderator variable of interest in the present study was adolescents' close friendships and social competence. Our findings suggest that for social and separation anxiety, close friendships may offer more of a buffer for immigrant children than for non-immigrant adolescents. For generalized anxiety, social competence seemed more important for non-immigrant adolescents than for their immigrant counterparts.

A lack of close friendships was more strongly correlated with social and separation anxiety for BAME adolescents than white native adolescents. This finding is consistent with Sirin et al. (2013) and Atzaba-Poria et al. (2004), who indicate that anxiety generally decreases with close peer friendships, because social support from intimate friendships acts as a buffer against anxiety for immigrant children. 
Close friendships are important to develop interpersonal intimacy and empathy, and can also conjure more positive self-esteem and psychological adjustments (Pontari and Glenn 2012). Accordingly, it is conceivable that the close friendships can be extremely important for immigrant adolescents, as they might need interpersonal relationships to navigate the new social setting and adjust.

However, the fact that close friendships were not significantly associated with social anxiety and separation anxiety for WNA is at odds with La Greca and Harrison (2005), who found that close friendships were associated with lower social anxiety $(r=-0.14)$. This discrepancy is not simply due to power, because the effect size observed in the current WNA sample was close to zero $(r=0.04)$. It could, however, reflect measurement differences: La Greca and Harrison measured positive (and negative) aspects of the person's best friend or romantic friend. In the current study, we measured the close friendship as a more general construct (i.e., not focussed on a particular friend). It is possible that for WNAs, forming close friendships with peers is not the major obstacle in their social development, whereas it might be their immigrant counterparts because they are in a new cultural setting.

Social competence was strongly correlated with anxiety measures for WNAs. Although these relationships were not significant in the IA group, this is most likely a power issue because the moderation analyses suggested that the size of the relationship between social competence and anxiety subscales was comparable for all except generalized anxiety (where the association was significantly stronger for WNAs). Harter (2012) argues that peer acceptance is an important part of forming self-identity for adolescents, which can reflect on individual social competence. In addition, strong social competence and more acceptance from peers is associated with peer crowds (La Greca and Harrison 2005), while being accepted in a crowd and general popularity contributes to lower social anxiety (La Greca et al. 2001). Our results are certainly consistent with this line of argument in WNA samples, although social competence appears a weaker correlate of general anxiety in immigrant adolescents. This finding does not reflect an overall lower level of social competence in immigrant adolescents (their mean was comparable to WNA), instead it reflects a difference in the relationship that social competence has with general anxiety in immigrant adolescents.

The design of the current study allows us to say nothing about cause and effect. Therefore, although it is tempting to conclude that the direction of the effects is that close friendships are more of a buffer against anxiety in BAME immigrant adolescents compared to natives, an equally valid interpretation is that high anxiety makes it harder for immigrant adolescents to form close friendships than natives. The clinical and practical implications of the findings are unchanged by which interpretation is true. The current findings suggest that facilitating close friendships in immigrant adolescents might be a particularly useful thing to do. Based on the first interpretation of the effects, facilitating close friendships will help to reduce social and separation anxiety, whereas attempts to enhance their social competence might be less effective. Based on the second interpretation, immigrant adolescents experiencing anxiety might find it hard to form close friendships, therefore, targeting activities that facilitate those friendships will help them.

The study had several methodological limitations. The findings of this study are a provisional piece of work based on a small sample in four areas in the UK; they are not representative of all immigrant adolescents in the UK, and not even of the four counties represented in this study. It is important that the parameters of the models are estimated in bigger samples, and the general pattern of results replicated. Further, the close friendship subscale in this study did not distinguish the quantity from the quality of close friendships.

Another possible problem highlighted during the recruitment period is the lower response rate from immigrant families compared with non-immigrant families. This difference could be a reflection of BAME parents' reluctance to bring their children to the study, due to their cultural beliefs and practices. Social stigma and discrimination may also be another contribution to the reluctance, particularly if the immigrant families are low-skilled workers or illegal immigrants (Blinder et al. 2011). 


\section{Conclusions}

Despite the growing number of BAME minority families in the UK, studies on BAME adolescent's peer friendship and social anxiety remain limited. This study extends research on social anxiety for immigrant adolescents. The findings of the present study suggest a different pattern of associations between close peer friendship and social anxiety across immigrant and native adolescents. This study indicates that, for the first generation of BAME immigrant adolescents, forming close friendships in a new setting may either be an important protective factor to prevent developing social and separation anxiety, or that having social anxiety might make it difficult for immigrant adolescents to form close friendships. If replicated in a larger sample, these findings have potential importance for policy makers and schools; for example, providing family systemic support for immigrant families and their children to integrate to the host country. For schools and communities, working together to facilitate activities such as buddy schemes to help adolescents foster close friendships might also reduce their social anxiety.

Further research is needed to find out if the culture of the immigrants impacts adolescents' ways of dealing with anxiety, as different cultural settings put in place different systems of enabling adolescents to meet their peers and learn or play together more than others. Finally, this study provided a one-time snapshot of BAME immigrant adolescents' peer friendship, and a longitudinal design comparing different groups in different settings will provide an important step to capturing the dynamic nature of how the peer friendships' impacts are associated with their social anxiety and wellbeing.

Author Contributions: This work is based on J.D.'s MSc dissertation which was supervised by A.P.F. The original research idea, methods, analysis and write-up were J.D.'s under A.P.F.'s supervision. A.P.F. conducted post hoc unplanned statistical analyses not central to the main aims of the paper (Bayes factors and robust versions of tests from the dissertation). The final manuscript was produced jointly. All authors have read and agreed to the published version of the manuscript.

Funding: This research received no external funding.

Conflicts of Interest: The authors declare no conflict of interest.

\section{References}

Louise, Ackers, and Helen Stalford. 2004. A Community for Children? Children, Citizenship and Internal Migration in the European Union. Aldershot: Ashgate.

Atzaba-Poria, Naama, Alison Pike, and Kirby Deater-Deckard. 2004. Do risk factors for problem behaviour act in a cumulative manner? An examination of ethnic minority and majority children through an ecological perspective. Journal of Child Psychology and Psychiatry 45: 707-18. [CrossRef] [PubMed]

Banerjee, Robin, Dawn Watling, and Marcella Caputi. 2011. Peer Relations and the Understanding of Faux Pas: Longitudinal Evidence for Bidirectional Associations. Child Development 82: 1887-905. [CrossRef] [PubMed]

Beesdo, Katja, Antje Bittner, Daniel S. Pine, Murray B. Stein, Michael Höfler, Roselind Lieb, and Hans-Ulrich Wittchen. 2007. Incidence of Social Anxiety Disorder and the Consistent Risk for Secondary Depression in the First Three Decades of Life FREE. Archives General Psychiatry 64: 903-91. [CrossRef] [PubMed]

Bhugra, Dinesh. 2004. Migration and mental health. Acta Psychiatrica Scandinavica 109: 243-58. [CrossRef] [PubMed]

Blinder, Scott, Martin Ruhs, and Carlos Vargas-Silva. 2011. Thinking Behind of the Numbers: Understanding Public Opinion on Immigration in Britain. Report. In The Migration Observatory. Oxford: Oxford University.

Cardoos, Stephanie L., and Stephen P. Hinshaw. 2011. Friendship as protection from peer victimization for girls with and without ADHD. Journal of Abnormal Child Psychology 39: 1035-45. [PubMed]

Cartwright-Hatton, Sam, Deb McNally, Andy P. Field, Stewart Rust, Ben Laskey, and Clare Dixon. 2011. A New Parenting-Based Group Intervention for Young Anxious Children: Results of a Randomized Controlled Trial. Journal of the American Academy of Child E Adolescent Psychiatry 3: 242-351. 
Chorpita, Bruce F., Letitia Yim, Catherine Moffitt, Lori A. Umemoto, and Sarah E. Francis. 2000. Assessment of symptoms of DSM-IV anxiety and depression in children: A revised child anxiety and depression scale. Behaviour Research and Therapy 38: 835-55. [CrossRef]

Dienes, Zoltan. 2014. Using Bayes to get the most out of non-significant results. Frontiers in Psychology 5. [CrossRef] Festa, Candice C., and Golda S. Ginsburg. 2011. Parental and Peer Predictors of Social Anxiety in Youth. Child Psychiatry \& Human Development 42: 291-306.

Hao, Lingxin, and Han S. Woo. 2012. Distinct Trajectories in the Transition to Adulthood: Are Children of Immigrants Advantaged? Child Development 83: 1623-39. [CrossRef]

Harker, Kathryn. 2001. Immigrant generation, assimilation, and adolescent psychological well-being. Journal of Social Forces 79: 969-1004.

Harter, Susan. 2012. Self-Perceptıon Profile for Adolescents: Manual and Questıonnaires (Revised). Denver: University of Denver.

Hayes, Andrew F. 2013. Introduction to Mediation, Moderation and Conditional Process Analysis: A Regression-Based Approach. New York: Guilford Publication.

Huang, Keng-Yen, Esther Calzada, Sabrina Cheng, and Laurie Miller Brotman. 2012. Physical and mental health disparities among young children of Asian immigrants. Journal of Pediatrics 160: 331-36. [PubMed]

Kessler, Ronald C., Patricia Berglund, Olga Demler, Robert Jin, Kathleen R. Merikangas, and Ellen E. Walters. 2005. Lifetime prevalence and age-of-onset distributions of DSM-IV disorders in the National Comorbidity Survey Replication. Archives of General Psychiatry 6: 593-602.

Kovacev, Lydia, and Rosalyn Shute. 2004. Acculturation and social support in relation to psychosocial adjustment of adolescent refugees resettled in Australia. International Journal of Behavioural Development 28: 259-67. [CrossRef]

La Greca, Annette M., and Hannah Moore Harrison. 2005. Adolescent peer relations, Friendships and romantic relationships: Do they predict social anxiety and depression? Journal of Clinical Child and Adolescent Psychology 34: 49-61. [PubMed]

La Greca, Annette M., Mitchell J. Prinstein, and Michael D. Fetter. 2001. Adolescent peer crowd affiliation: Linkages with health-risk behaviours and close friendships. Journal of Paediatric Psychology 26: 131-43. [CrossRef]

Lee, Susan. 2019. Does identity matter for adaptation? The influence of acculturation identity on the well-being and delinquency of adolescent students in Europe. Journal of Youth Studies, 1-21. [CrossRef]

Moitra, Ethan, Roberto Lewis-Fernández, Robert L. Stout, Erica Angert, Risa B. Weisberg, and Martin B. Keller. 2014. Disparities in psychosocial functioning in a diverse sample of adults with anxiety disorders. Journal of Anxiety Disorders 28: 335-43.

Montgomery, Edith. 2008. Long-term effects of organized violence on young Middle Eastern refugees' mental health. Social Science and Medicine 67: 1596-603.

Morey, Richard D., and Jeffrey N. Rouder. 2014. Bayes Factor: Computation of Bayes factors for Common Designs. R Package (Version 0.9.7). Available online: http://CRAN.R-project.org/package=BayesFactor (accessed on 1 May 2020).

National Institute for Health and Care Excellence. 2013. Social Anxiety Disorder: Recognition, Assessment and Treatment]. [CG 159]. London: National Institute for Health and Care Excellence.

Perreira, Krista M., and India J. Ornelas. 2011. The physical and psychological well-being of immigrant children. The Future of Children 21: 195-218. [CrossRef]

Pina, Armando A., and Wendy K. Silverman. 2004. Clinical Phenomenology, Somatic Symptoms, and Distress in Hispanic/Latino and European American Youths with Anxiety Disorders. Journal of Clinical Child E Adolescent Psychology 33: 227-36.

Pontari, Beth A., and Ellen J. Glenn. 2012. Engaging in less protective self-presentation: The effects of a friend's presence on the socially anxious. Basic and Applied Social Psychology 34: 516-26.

Qin, Desirée Boalian, Niobe Way, and Preetika Mukherjee. 2008. The other side of the model minority story: The familial and peer challenges faced by Chinese American adolescents. Youth E Society 39: 480-506.

R Core Team. 2015. R: A Language and Environment for Statistical Computing. Vienna: R Foundation for Statistical Computing, Available online: http://www.R-project.org (accessed on 1 May 2020).

Rapee, Ronald M., and Susan H. Spence. 2004. The aetiology of social phobia: Empirical evidence and an initial model. Clinical Psychology Review 24: 737-67. [CrossRef] [PubMed] 
Rouder, Jeffrey N., Paul L. Speckman, Dongchu Sun, Richard Donald Morey, and G. J. Iverson. 2009. Bayesian t tests for accepting and rejecting the null hypothesis. Psychonomic Bulletin E Review 16: 225-37. [CrossRef]

Seaton, Eleanor K., Tiffany Yip, and Robert M. Sellers. 2009. A longitudinal examination of racial identity and racial discrimination among African American adolescents. Child Development 80: 406-17. [CrossRef] [PubMed]

Sirin, Selcuk R., Taveeshi Gupta, Patrice Ryce, Dalal Katsiaficas, Carola Suárez-Orozco, and Lauren Rogers-Sirin. 2013. Understanding the role of social support in trajectories of mental health symptoms for immigrant adolescents. Journal of Applied Developmental Psychology 34: 199-207. [CrossRef]

Tsai, Jeanne L., Yu-Wen Ying, and Peter A. Lee. 2000. The meaning of "being Chinese" and "being American": Variation among Chinese-American young adults. Journal of Cross-Culture Psychology 31: 302-32. [CrossRef]

Van Zalk, Nejra, and Maarten Van Zalk. 2014. The Importance of Perceived Care and Connectedness with Friends and Parents for Adolescent Social Anxiety. Journal of Personality 83: 346-60. [CrossRef]

Woolcock, Michael, and Deeta Narayan. 2000. Social capital: Implications for development theory, research, and policy. The World Bank Research Observer 15: 225-49. [CrossRef]

(C) 2020 by the authors. Licensee MDPI, Basel, Switzerland. This article is an open access article distributed under the terms and conditions of the Creative Commons Attribution (CC BY) license (http://creativecommons.org/licenses/by/4.0/). 